\title{
Enhanced Recovery From Organic-Rich Shales Through Carbon Dioxide Injection: Molecular-Level Investigation
}

Saad Alafnan, Yusuf Falola, Osamah Al Mansour, Khalid AlSamadony, Abeeb Awotunde, Murtada Aljawad 
Appendix

Table 1. Fugacity data for pure $\mathrm{CH}_{4}$ and pure $\mathrm{CO}_{2}$ at $350 \mathrm{~K}$.

\begin{tabular}{|c|c|c|}
\hline $\mathrm{P}$ (Mpa) & $\mathrm{CH}_{4}$ Fug (Mpa) & $\mathrm{CO}_{2}$ Fug (Mpa) \\
\hline 2.067 & 2.028 & 1.930 \\
\hline 3.445 & 3.340 & 3.072 \\
\hline 6.890 & 6.494 & 5.466 \\
\hline 10.335 & 9.503 & 7.279 \\
\hline 20.670 & 18.056 & 10.680 \\
\hline 41.340 & 35.842 & 16.299 \\
\hline
\end{tabular}

Table 2. Adsorption data for the pure $\mathrm{CH}_{4}$ and $\mathrm{CO}_{2}$ at $350 \mathrm{~K}$.

\begin{tabular}{|c|c|c|}
\hline $\mathbf{P}$ (MPa) & $\mathrm{CO}_{\mathbf{2}}$ (molecules) & $\mathbf{C H}_{\mathbf{4}}$ (molecules) \\
\hline 2.067 & 53.39 & 29.83 \\
\hline 3.445 & 59.44 & 36.41 \\
\hline 6.890 & 65.78 & 45.97 \\
\hline 10.335 & 68.91 & 50.87 \\
\hline 20.67 & 72.01 & 58.37 \\
\hline 41.340 & 74.74 & 64.77 \\
\hline
\end{tabular}


Table 3. Fugacity data for the binary mixture of $\mathrm{CH}_{4}-\mathrm{CO}_{2}$ at $350 \mathrm{~K}$.

\begin{tabular}{|c|c|c|c|c|c|}
\hline & $\mathbf{P}(\mathrm{MPa})$ & $\mathrm{CH}_{4}$ phi-f & $\mathrm{CO}_{2}$ phi-f & $\mathrm{CH}_{4}$ Fug (MPa) & $\mathrm{CO}_{2}$ Fug (MPa) \\
\cline { 2 - 6 } & 2.067 & 0.982 & 0.954 & 1.624 & 0.394 \\
\cline { 2 - 6 } $\mathbf{Y}_{\mathrm{CH} 4}=\mathbf{0 . 8}$ & 3.445 & 0.971 & 0.925 & 2.675 & 0.637 \\
\cline { 2 - 6 } & 6.890 & 0.945 & 0.856 & 5.207 & 1.179 \\
\cline { 2 - 6 } & 10.335 & 0.923 & 0.794 & 7.630 & 1.641 \\
\cline { 2 - 6 } & 20.670 & 0.880 & 0.654 & 14.556 & 2.705 \\
\cline { 2 - 6 } & 31.050 & 0.868 & 0.576 & 21.552 & 3.576 \\
\hline $\mathbf{Y}_{\mathrm{CH} 4}=\mathbf{0 . 6}$ & 2.067 & 0.984 & 0.950 & 1.220 & 0.785 \\
\cline { 2 - 6 } & 3.445 & 0.974 & 0.917 & 2.013 & 1.264 \\
\cline { 2 - 6 } & 6.890 & 0.952 & 0.841 & 3.935 & 2.318 \\
\cline { 2 - 6 } & 10.335 & 0.934 & 0.772 & 5.792 & 3.191 \\
\cline { 2 - 6 } & 20.670 & 0.904 & 0.615 & 11.214 & 5.087 \\
\cline { 2 - 6 } & 31.050 & 0.900 & 0.529 & 16.767 & 6.574 \\
\hline \multirow{5}{*}{$\mathbf{Y}_{\mathrm{CH} 4}=\mathbf{0 . 4}$} & 2.067 & 0.987 & 0.946 & 0.816 & 1.174 \\
\cline { 2 - 6 } & 3.445 & 0.980 & 0.912 & 1.350 & 1.885 \\
\cline { 2 - 6 } & 6.890 & 0.965 & 0.830 & 2.659 & 3.430 \\
\cline { 2 - 6 } & 10.335 & 0.956 & 0.754 & 3.953 & 4.677 \\
\cline { 2 - 6 } & 20.670 & 0.957 & 0.582 & 7.915 & 9.214 \\
\cline { 2 - 6 } & 31.050 & 0.970 & 0.492 & 12.049 & 9.159 \\
\hline
\end{tabular}


Table 4. Fugacity data for the binary mixture of $\mathrm{CH}_{4}-\mathrm{CO}_{2}$ at $420 \mathrm{~K}$.

\begin{tabular}{|c|c|c|c|c|c|}
\hline & $\mathbf{P}(\mathrm{MPa})$ & $\mathrm{CH}_{4}$ phi-f & $\mathrm{CO}_{2}$ phi-f & $\mathrm{CH}_{4}$ Fug $(\mathrm{MPa})$ & $\mathrm{CO}_{2}$ Fug (MPa) \\
\cline { 2 - 6 } & 2.067 & 0.994 & 0.978 & 1.643 & 0.404 \\
\cline { 2 - 6 } & 3.445 & 0.990 & 0.963 & 2.729 & 0.664 \\
\cline { 2 - 6 } $\mathbf{Y}_{\mathrm{CH} 4}=\mathbf{0 . 8}$ & 6.890 & 0.982 & 0.930 & 5.414 & 1.281 \\
\cline { 2 - 6 } & 10.335 & 0.976 & 0.900 & 8.072 & 1.859 \\
\cline { 2 - 6 } & 20.670 & 0.971 & 0.828 & 16.049 & 3.424 \\
\cline { 2 - 6 } & 31.050 & 0.981 & 0.784 & 24.369 & 4.867 \\
\hline \multirow{5}{*}{$\mathbf{Y}_{\mathrm{CH} 4}=0.6$} & 2.067 & 0.995 & 0.975 & 1.234 & 0.806 \\
\cline { 2 - 6 } & 3.445 & 0.992 & 0.959 & 2.050 & 1.322 \\
\cline { 2 - 6 } & 6.890 & 0.986 & 0.922 & 4.076 & 2.540 \\
\cline { 2 - 6 } & 10.335 & 0.982 & 0.887 & 6.090 & 3.668 \\
\cline { 2 - 6 } & 20.670 & 0.982 & 0.805 & 12.184 & 6.657 \\
\cline { 2 - 6 } & 31.050 & 0.998 & 0.753 & 18.600 & 9.346 \\
\hline \multirow{5}{*}{$\mathbf{Y}_{\mathrm{CH} 4}=\mathbf{0 . 4}$} & 2.067 & 0.997 & 0.973 & 0.824 & 1.207 \\
\cline { 2 - 6 } & 3.445 & 0.995 & 0.956 & 1.371 & 1.976 \\
\cline { 2 - 6 } & 6.890 & 0.993 & 0.916 & 2.736 & 3.785 \\
\cline { 2 - 6 } & 10.335 & 0.992 & 0.878 & 4.103 & 5.445 \\
\cline { 2 - 6 } & 20.670 & 1.005 & 0.787 & 8.310 & 9.763 \\
\cline { 2 - 6 } & 31.050 & 1.032 & 0.728 & 12.821 & 13.564 \\
\hline
\end{tabular}

